\title{
Supersymmetry Searches in ATLAS
}

\section{Elena Romero Adam}

(IFIC - CSIC / Universidad de Valencia)

on behalf of the ATLAS Collaboration

August 25, 2015

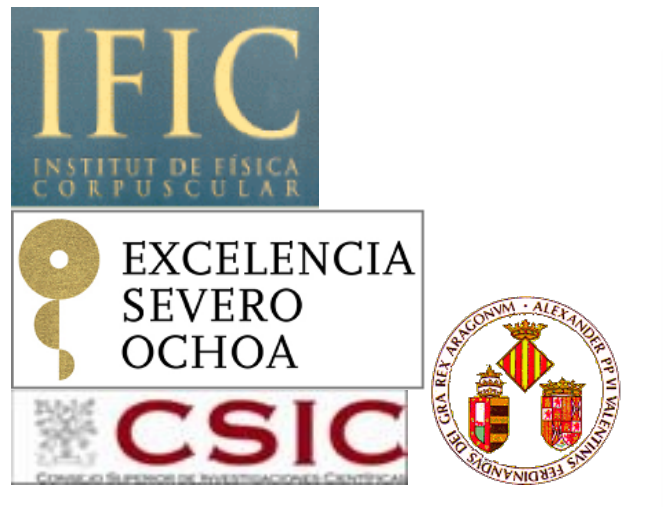

$17^{\text {th }}$ Lomonosov Conference on Elementary Particle Physics 


\section{Outline}

- Introduction

- Supersymmetry

- ATLAS SUSY Search Strategy \& Classification

- Strong Production Searches

- Searches for direct pair production of $3^{\text {rd }}$ generation squarks

- Electroweak Production Searches

- RPV Searches

- What's next? 


\section{Introduction}




\section{Supersymmetry (SUSY)}

\section{Standard particles}

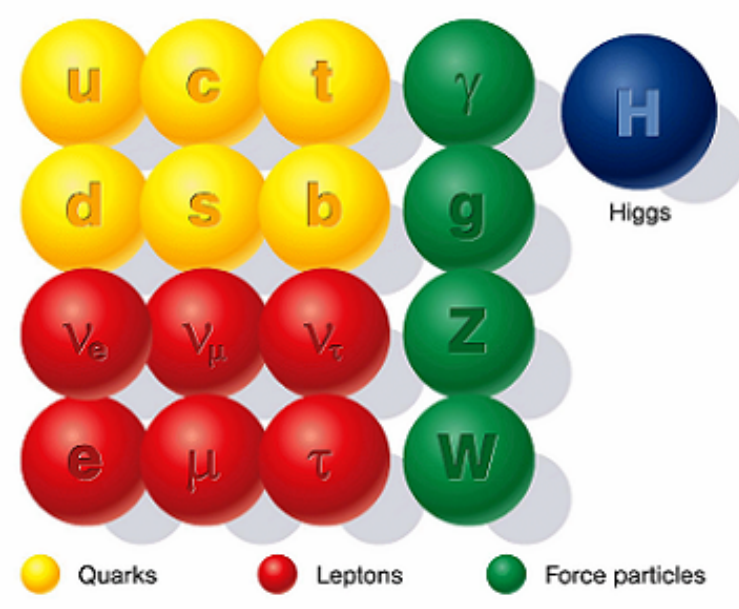

\section{SUSY particles}

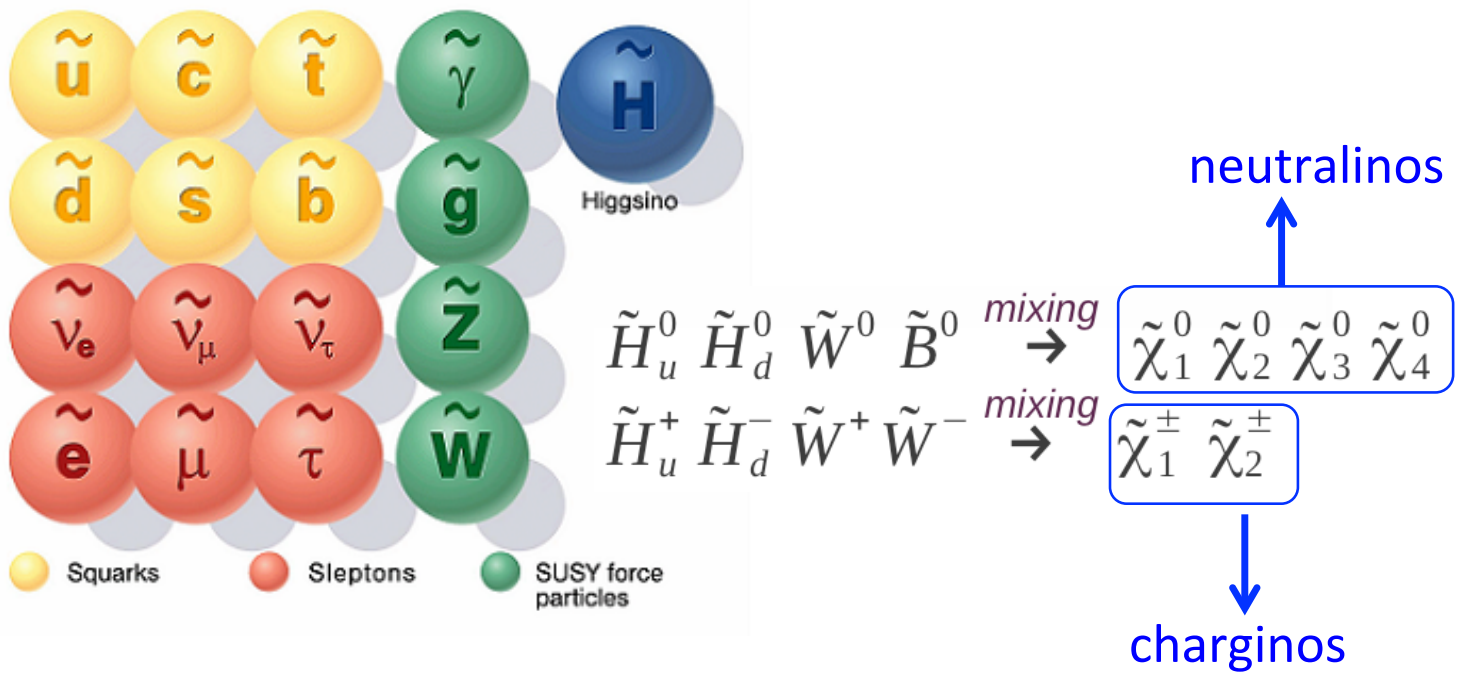

- $\quad$ SUSY = global symmetry between fermions \& bosons

- $\quad$ all SM particles have SUSY-partners with spin difference of $\pm 1 / 2$

- $\quad$ SUSY has to be a broken symmetry (superpartners heavier than SM particles)

- Theoretical motivation

- Higgs mass stabilization against loop corrections (fine-tuning problem)

- unification of gauge couplings at single scale

- dark matter candidate:

Lightest supersymmetric particle (LSP) 


\section{ATLAS SUSY Search Strategy}

\section{SUSY events in our data?}

- Define Signal Region(s) (SR): set of cuts (on EtMiss, jet multiplicity, etc) that favour signal over background

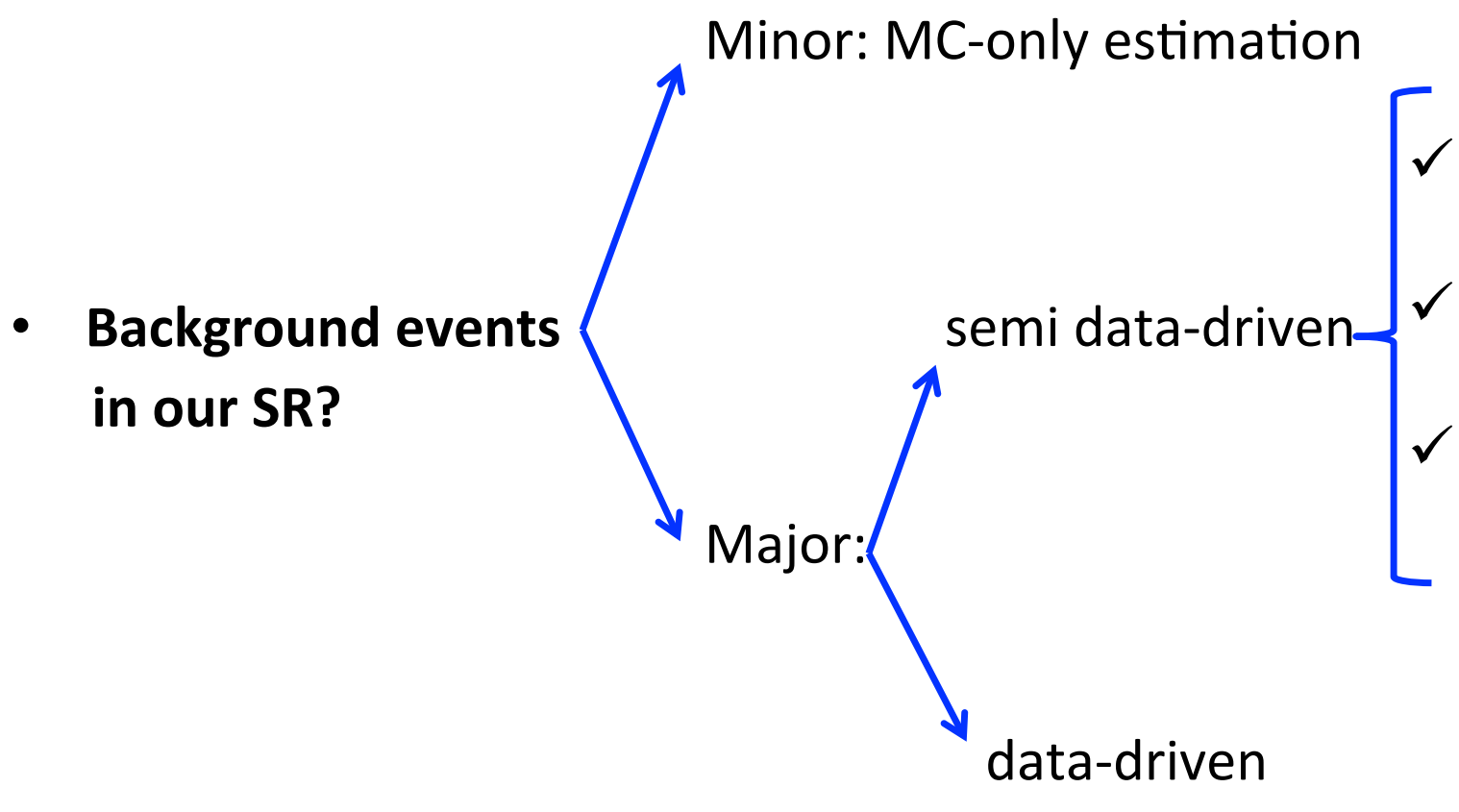

- Discovery/Exclusion fit:

- CLs exclusion limits or discovery

Define Control Regions (CR): negligible signal

$\checkmark$ Normalise MC to match data in CR (obtain transfer factor)

$\checkmark$ Apply transfer factor to MC estimation of SR bkg

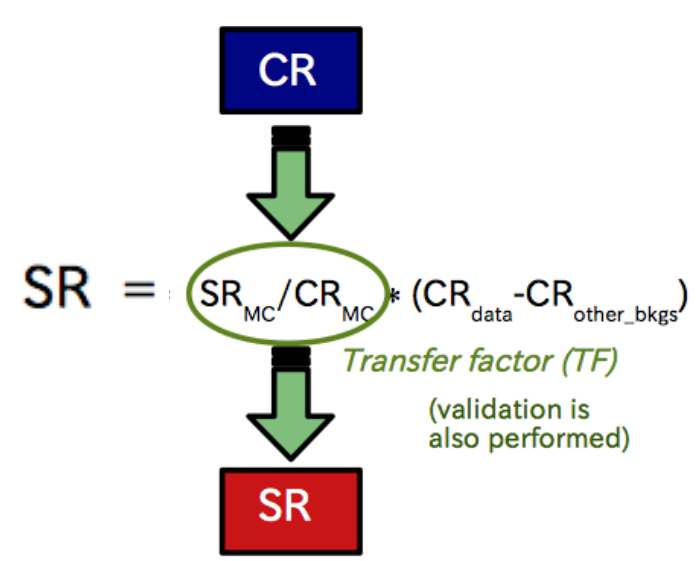




\section{Classifying SUSY searches}

Searches split by production process...

- Strong production

- squarks (1st \& $2^{\text {nd }}$ gen.) \& gluinos

- $3^{\text {rd }}$ generation production

- targeting stops and sbottoms

- Electroweak production

- targeting electroweak gauginos \& sleptons

\section{... and by final state characteristics}

- R-parity conserving (RPC) SUSY:

- neutral stable LSP

- sparticles produced in pairs

- large MET

- R-parity violating (RPV) SUSY:

- LSP decays into SM particles $=>$ (in some cases) single sparticle production

- Long-Lived particles

R-parity:

$$
R=(-1)^{3 B+L+2 S}
$$

- +1 for SM particles

- -1 for SUSY particles 


\section{Strong Production Searches}

- 95\% CL exclusion limits from: arXiv:1507.05525 (SUSY strong prod. summary paper)

- Only a few examples displayed:

Simplified model: $\tilde{q}-\tilde{q} \rightarrow q q W W \tilde{\chi}_{1}^{0} \widetilde{\chi}_{1}^{0}$

$$
V s=8 \mathrm{TeV}, \mathrm{L}=20 \mathrm{fb}^{-1}
$$

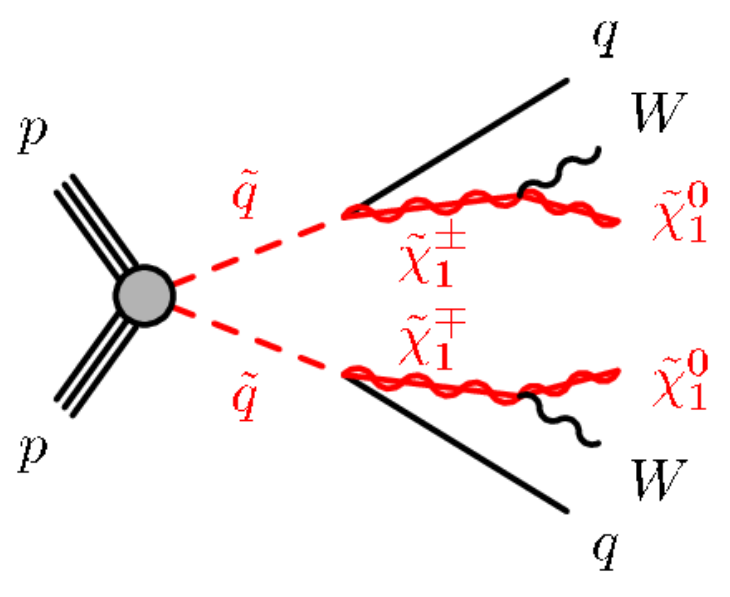

- 0-lepton + 1-lepton combination extends the reach

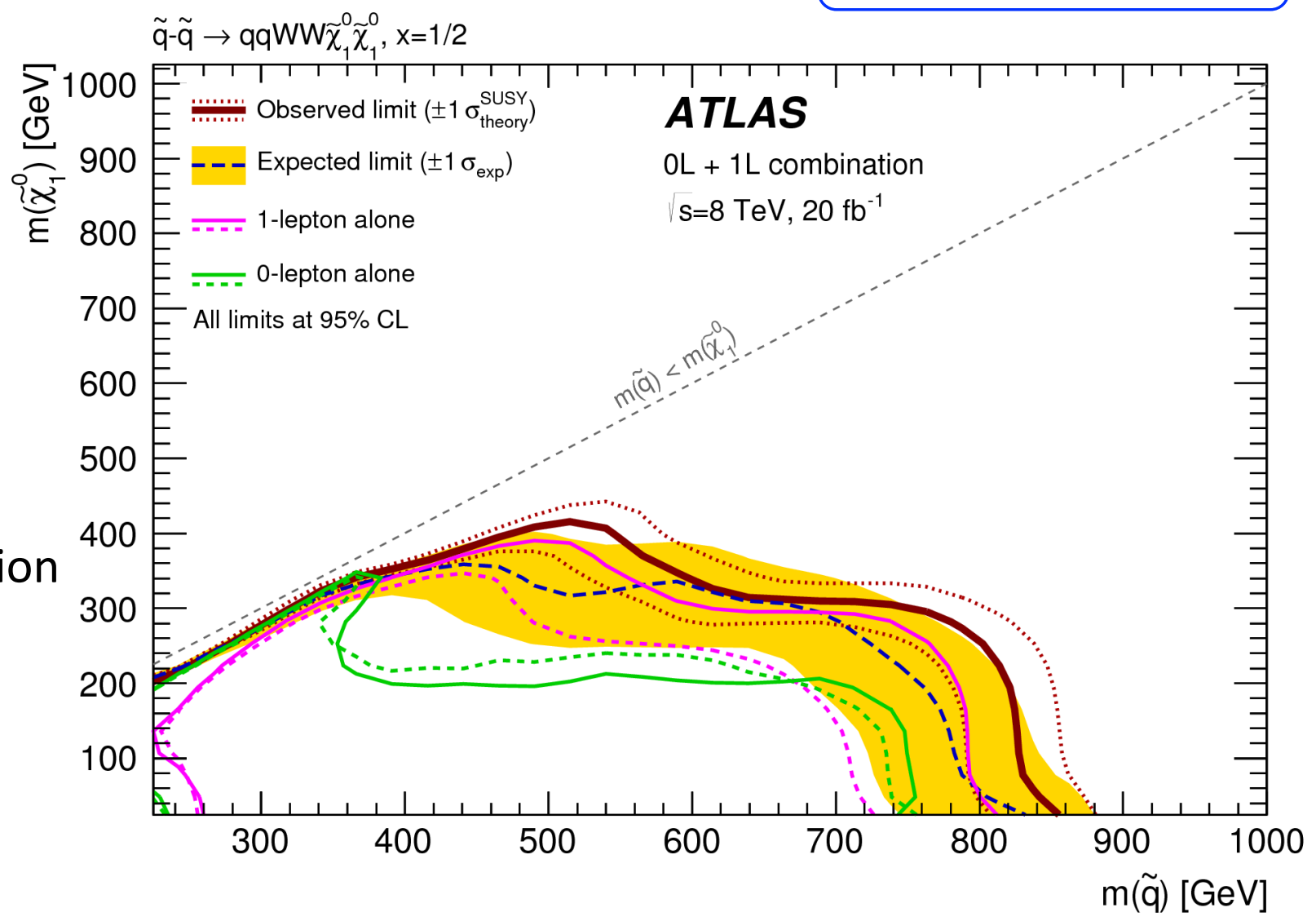




\section{Strong Production Searches}

- 95\% CL exclusion limits from: arXiv:1507.05525 (SUSY strong prod. summary paper)

- Only a few examples displayed:

\section{MSUGRA/CMSSM}

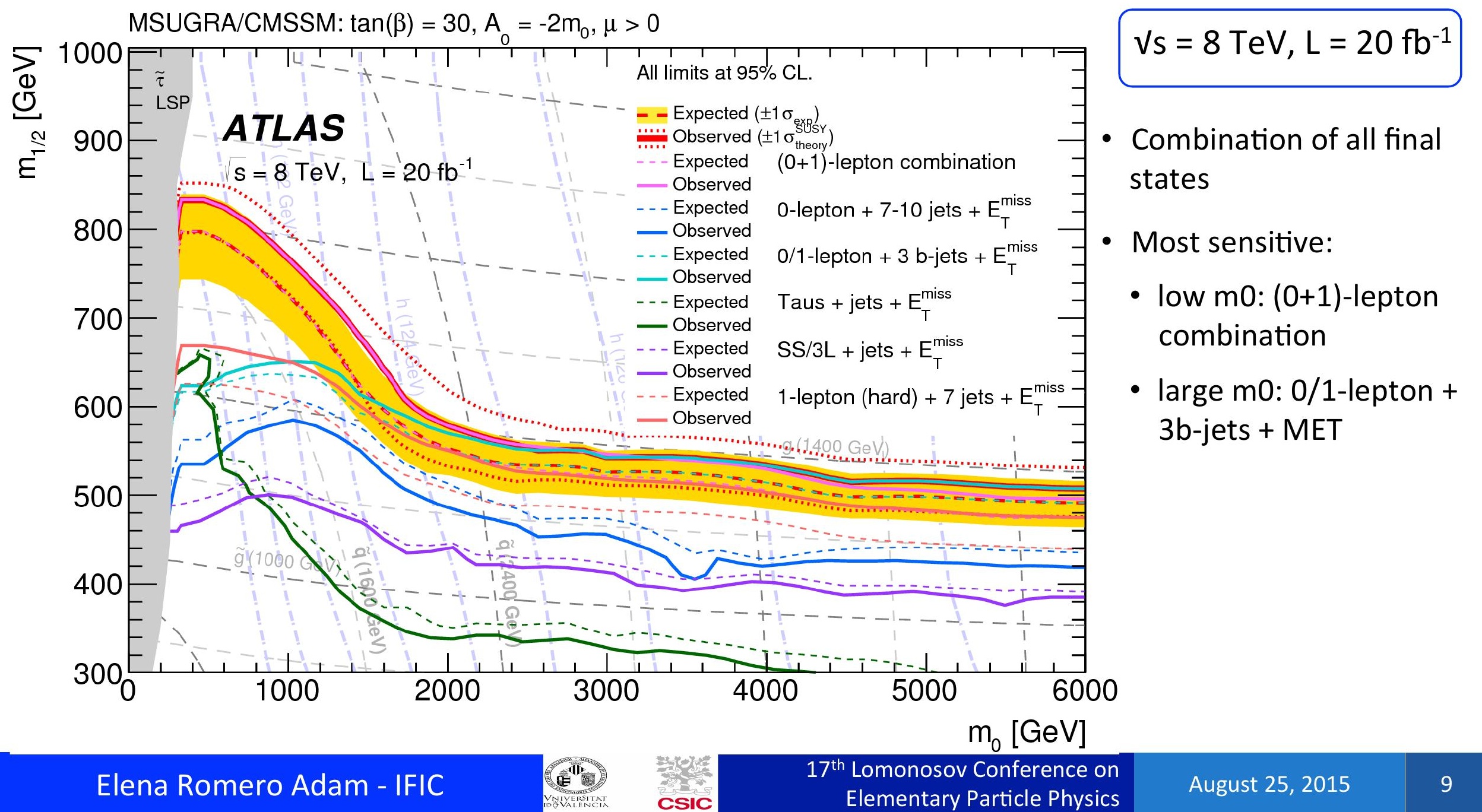




\section{Strong Production Searches}

\section{$3 \sigma$ excess in the Z+MET search: arXiv:1503.03290}

Events with: $Z->e^{+} e^{-} / \mu^{+} \mu^{-}+\geq 2$ jets $+E_{T}$ miss

- Data-driven methods for all major backgrounds

- thoroughly cross-checked with other methods

(including data-driven, semi data-driven and MC)

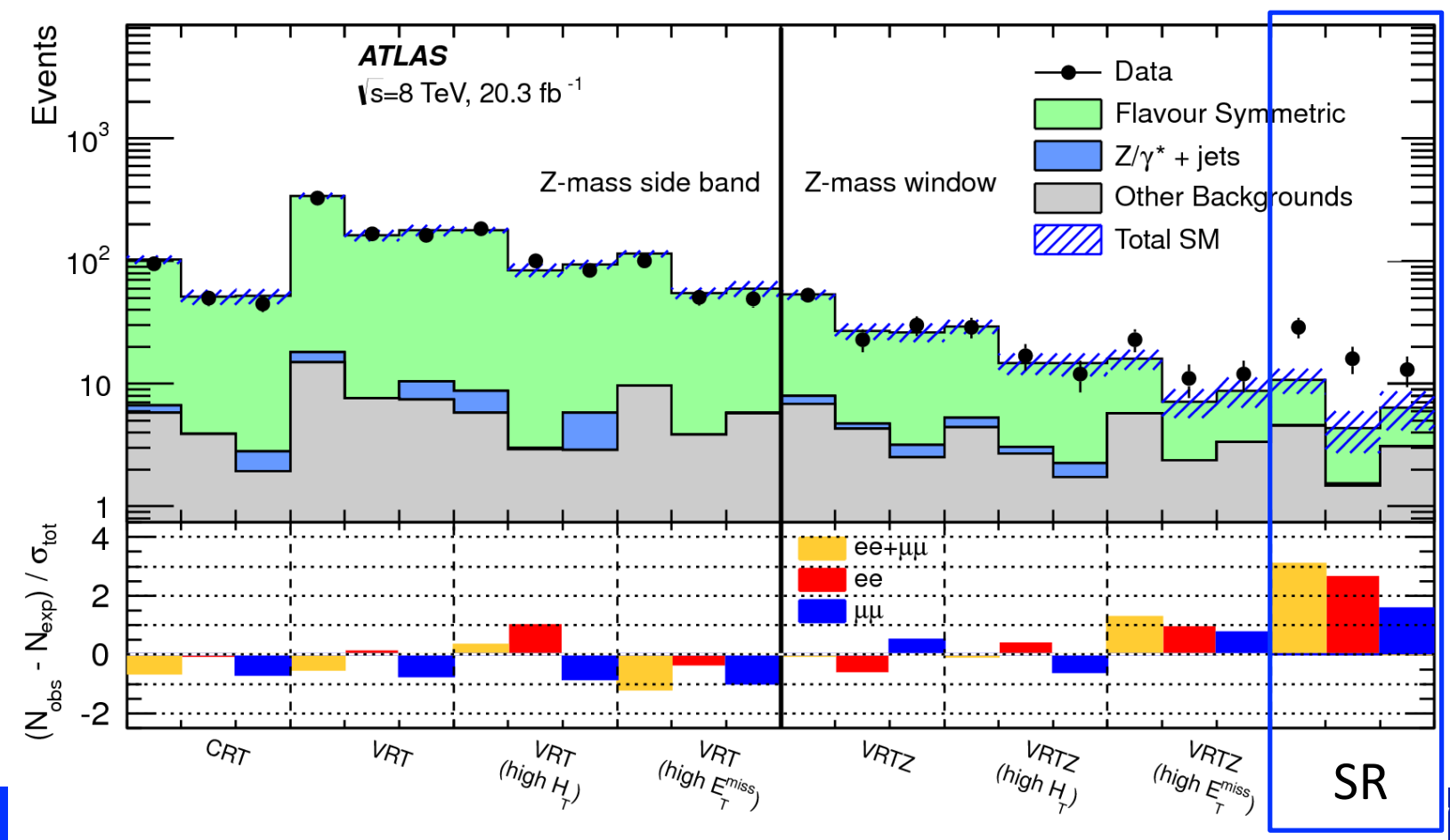




\section{Strong Production Searches}

\section{$3 \sigma$ excess in the $Z+M E T$ search: arXiv: 1503.03290}

Events with: $Z->\mathrm{e}^{+} \mathrm{e}^{-} / \mu^{+} \mu^{-}+\geq 2$ jets $+\mathrm{E}_{\mathrm{T}}$ miss

- Data-driven methods for all major backgrounds

- thoroughly cross-checked with other methods (including data-driven, semi data-driven and MC)

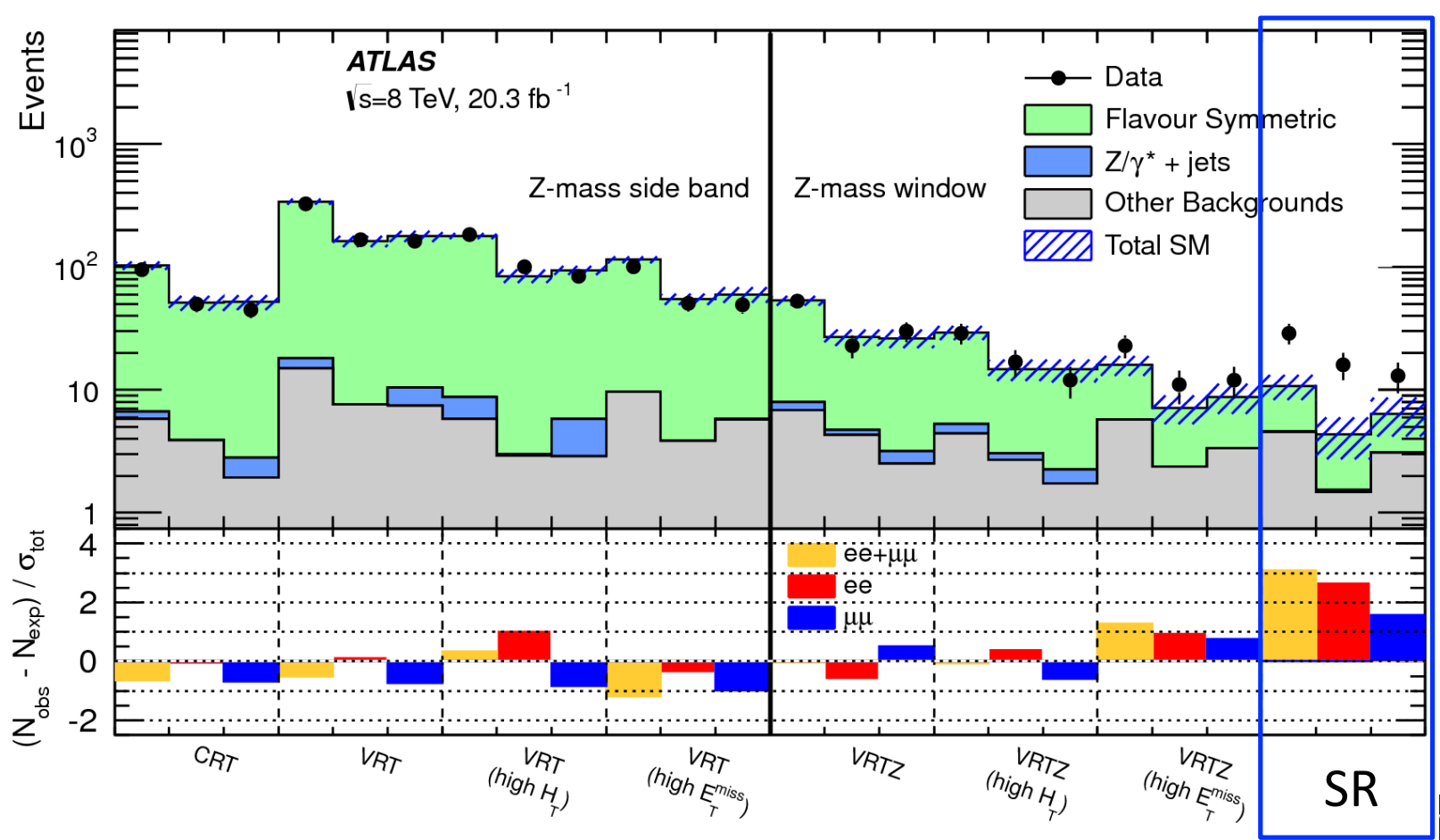

$\mathrm{Vs}=8 \mathrm{TeV}, \mathrm{L}=20 \mathrm{fb}^{-1}$

\begin{tabular}{|ccc|c|}
\hline SR & Expected & Observed & Signif. \\
\hline ee & $4.2 \pm 1.6$ & 16 & $3 \sigma$ \\
\hline$\mu \mu$ & $6.4 \pm 2.2$ & 13 & $1.7 \sigma$ \\
\hline ee $+\mu \mu$ & $10.6 \pm 3.2$ & 29 & $3 \sigma$ \\
\hline
\end{tabular}

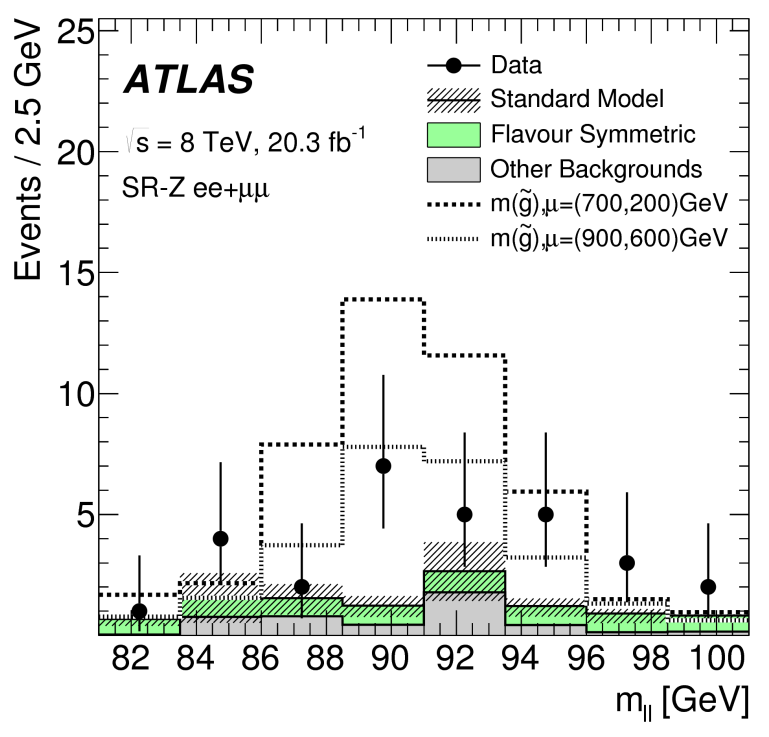

Conference on Particle Physics 


\section{Strong Production Searches}

\section{$3 \sigma$ excess in the Z+MET search: arXiv:1503.03290}

Events with: $Z->e^{+} e^{-} / \mu^{+} \mu^{-}+\geq 2$ jets $+E_{T}$ miss

$$
V s=8 \mathrm{TeV}, \mathrm{L}=20 \mathrm{fb}^{-1}
$$

- 95\% CL exclusion limits set for target model GGM

- Limits weaker than expected due to the excess in data
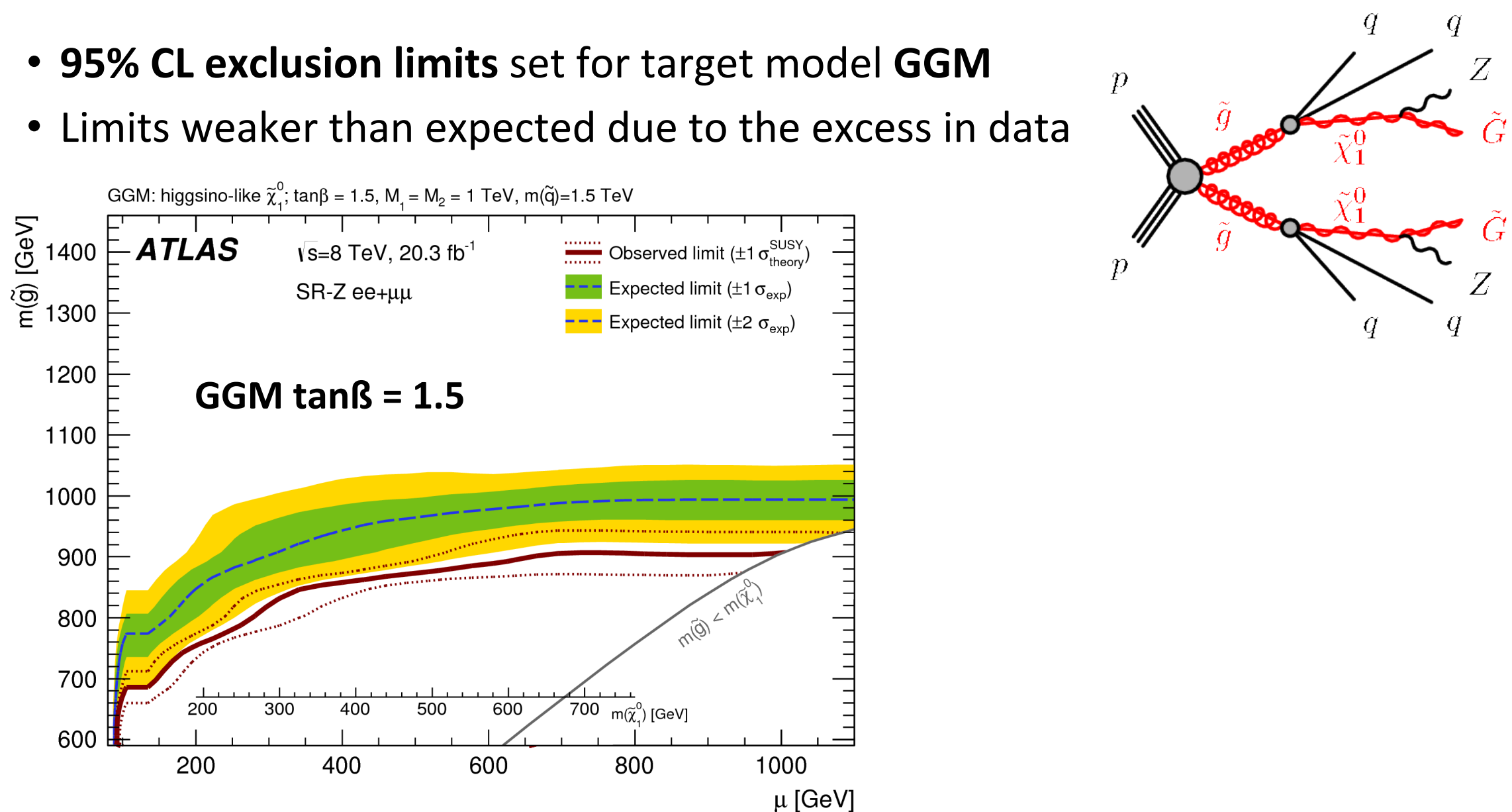


\section{Strong Production Searches}

\section{$3 \sigma$ excess in the Z+MET search: arXiv:1503.03290}

Events with: $Z->\mathrm{e}^{+} \mathrm{e}^{-} / \mu^{+} \mu^{-}+\geq 2$ jets $+\mathrm{E}_{\mathrm{T}}$ miss

$$
\sqrt{ } \mathrm{s}=8 \mathrm{TeV}, \mathrm{L}=20 \mathrm{fb}^{-1}
$$

- 95\% CL exclusion limits set for target model GGM

- Limits weaker than expected due to the excess in data

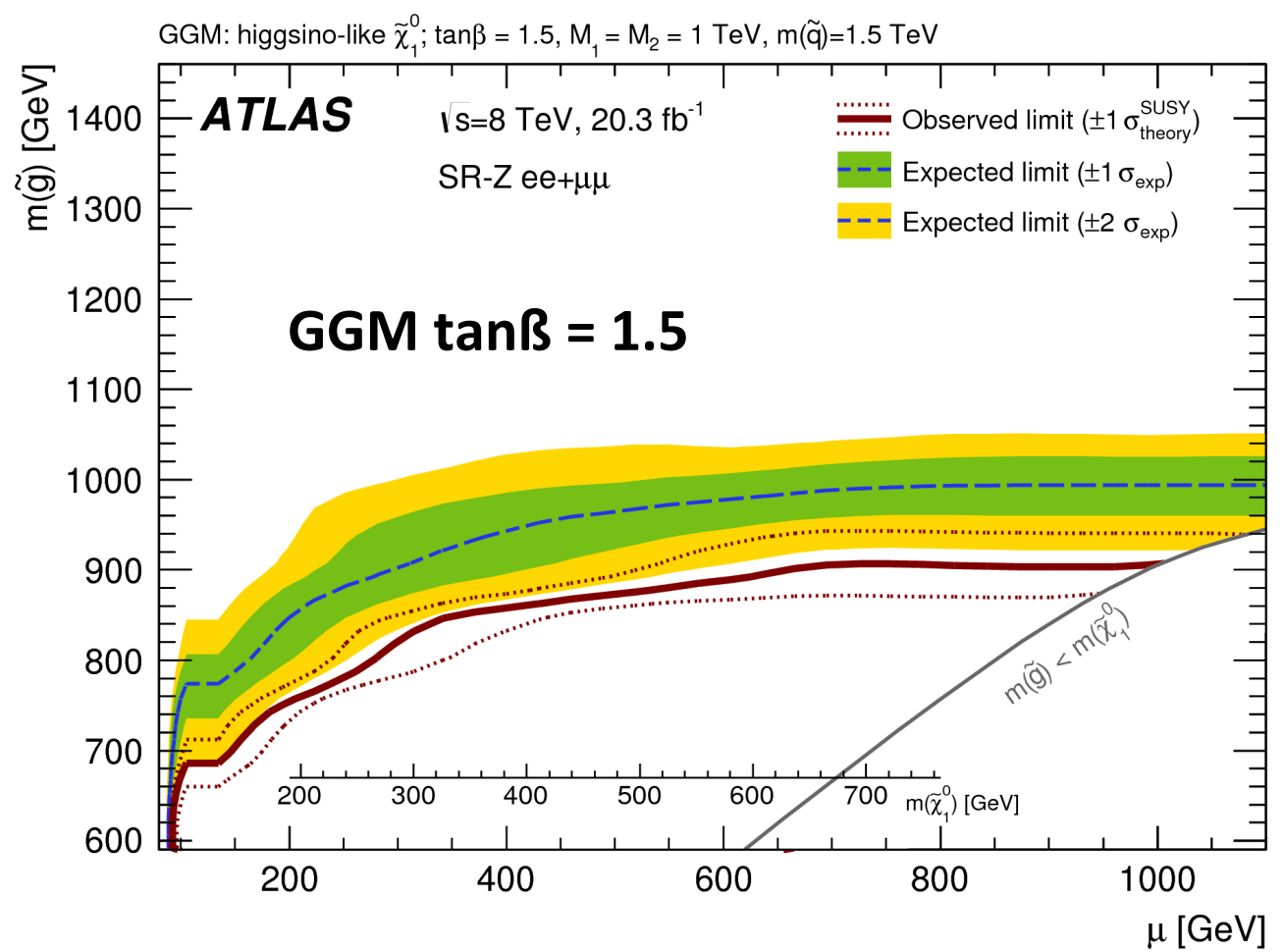




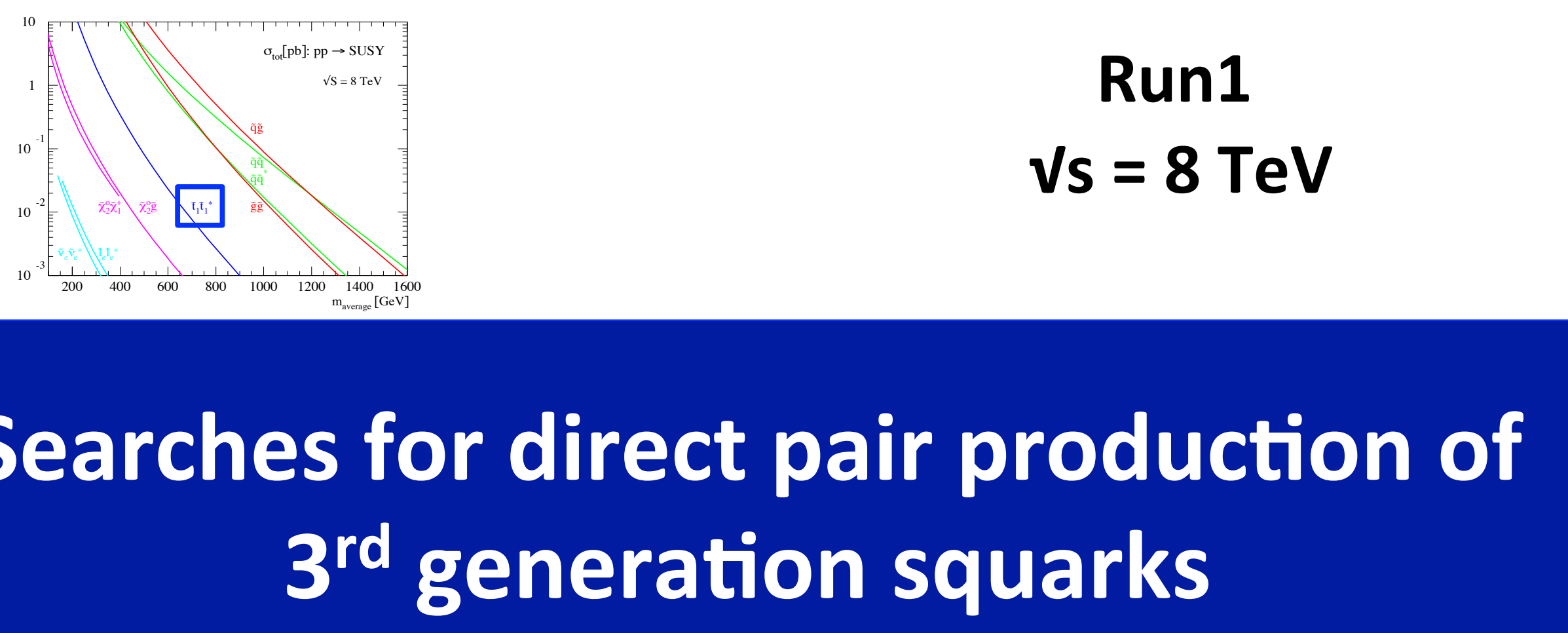

\section{Searches for direct pair production of} $3^{\text {rd }}$ generation squarks

- Large range of final states has been investigated

- Results interpreted in the context of many models

- Only a few examples shown in this talk 


\section{Direct pair production of $3^{\text {rd }}$ generation}

- 95\% CL exclusion limits from: arXiv:1506.08616 ( $3^{\text {rd }}$ gen. $\tilde{q}$ direct prod. summary paper)

- Example: Stop decays with no charginos in the decay chain

Stop decay modes defined mainly by:

$\Delta \mathrm{m}$ (stop, neutralino)

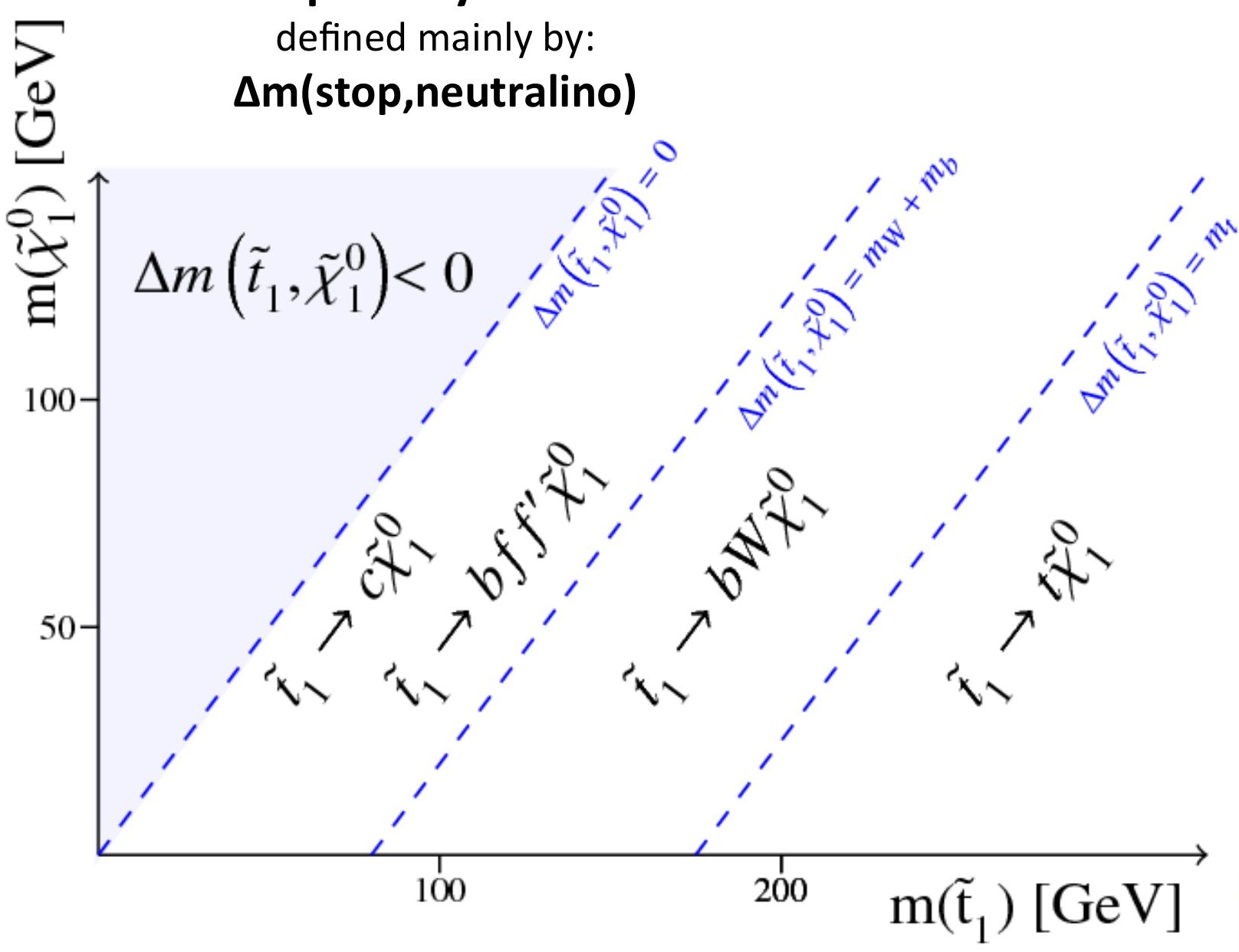




\section{Direct pair production of $3^{\text {rd }}$ generation}

- 95\% CL exclusion limits from: arXiv:1506.08616 (3rd gen. $\tilde{q}$ direct prod. summary paper)

- Example: Stop decays with no charginos in the decay chain

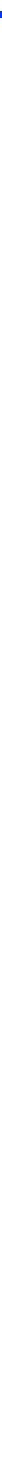




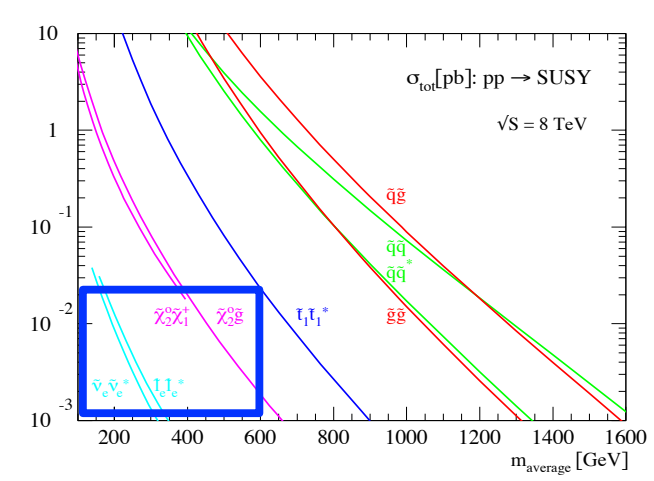

\section{Run1 \\ Vs $=8 \mathrm{TeV}$}

\section{Electroweak Production Searches}




\section{Electroweak Production Searches}

- Small cross-sections but:

$$
V s=8 \mathrm{TeV}, \mathrm{L}=20 \mathrm{fb}^{-1}
$$

- Clean multi-lepton final states

- Low hadronic activity

- Searches using e, $\mu, \tau$

Summary plot showing 95\% CL exclusion limits for SM bosons mediated decays

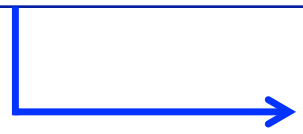

Publication references shown in the plot

ATLAS Preliminary $20.3 \mathrm{fb}^{-1}$, $\sqrt{s}=8 \mathrm{TeV}$

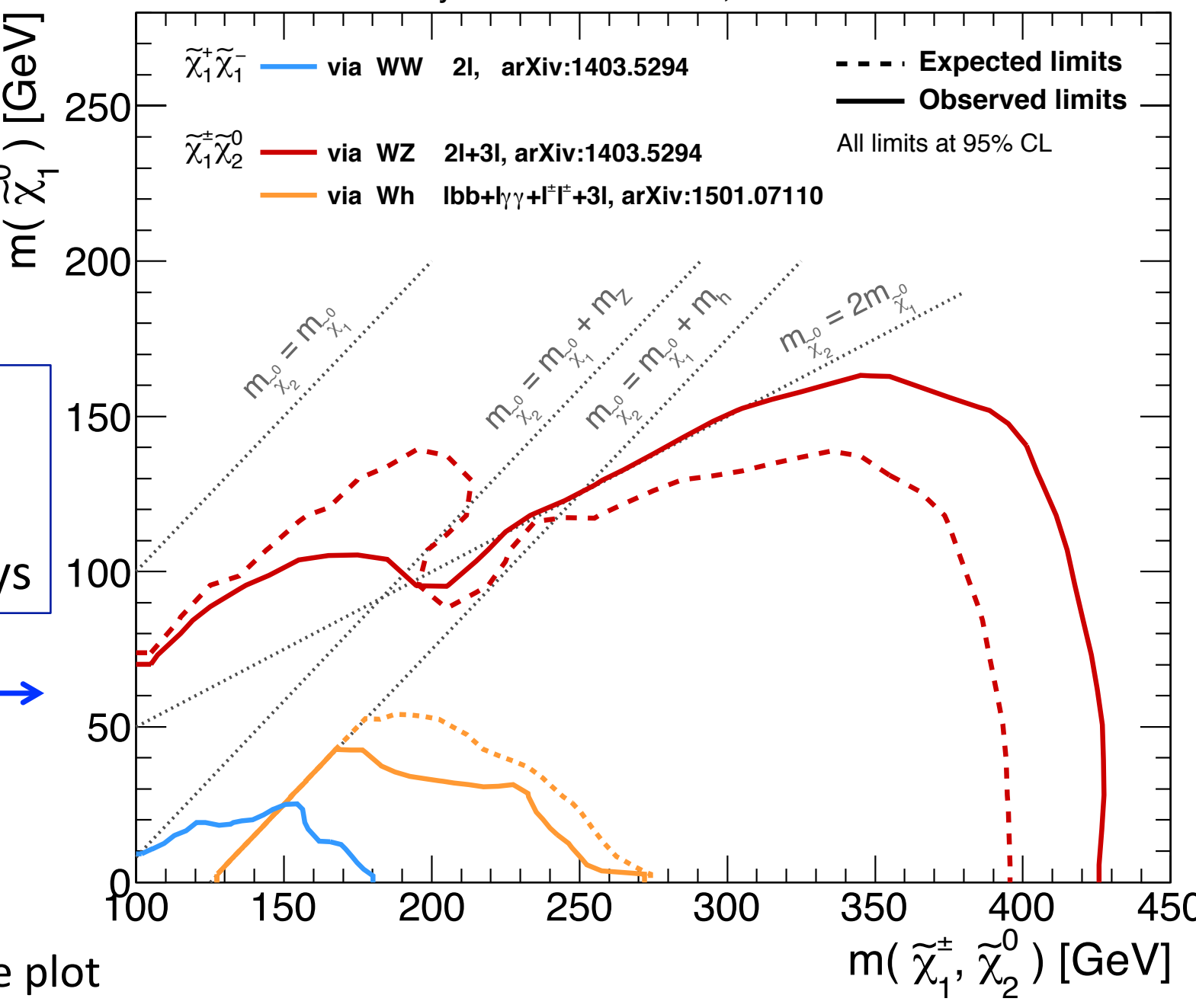




\section{Electroweak Production Searches}

- Small cross-sections but:

$$
V s=8 \mathrm{TeV}, \mathrm{L}=20 \mathrm{fb}^{-1}
$$

- Clean multi-lepton final states

- Low hadronic activity

- Searches using e, $\mu, \tau$

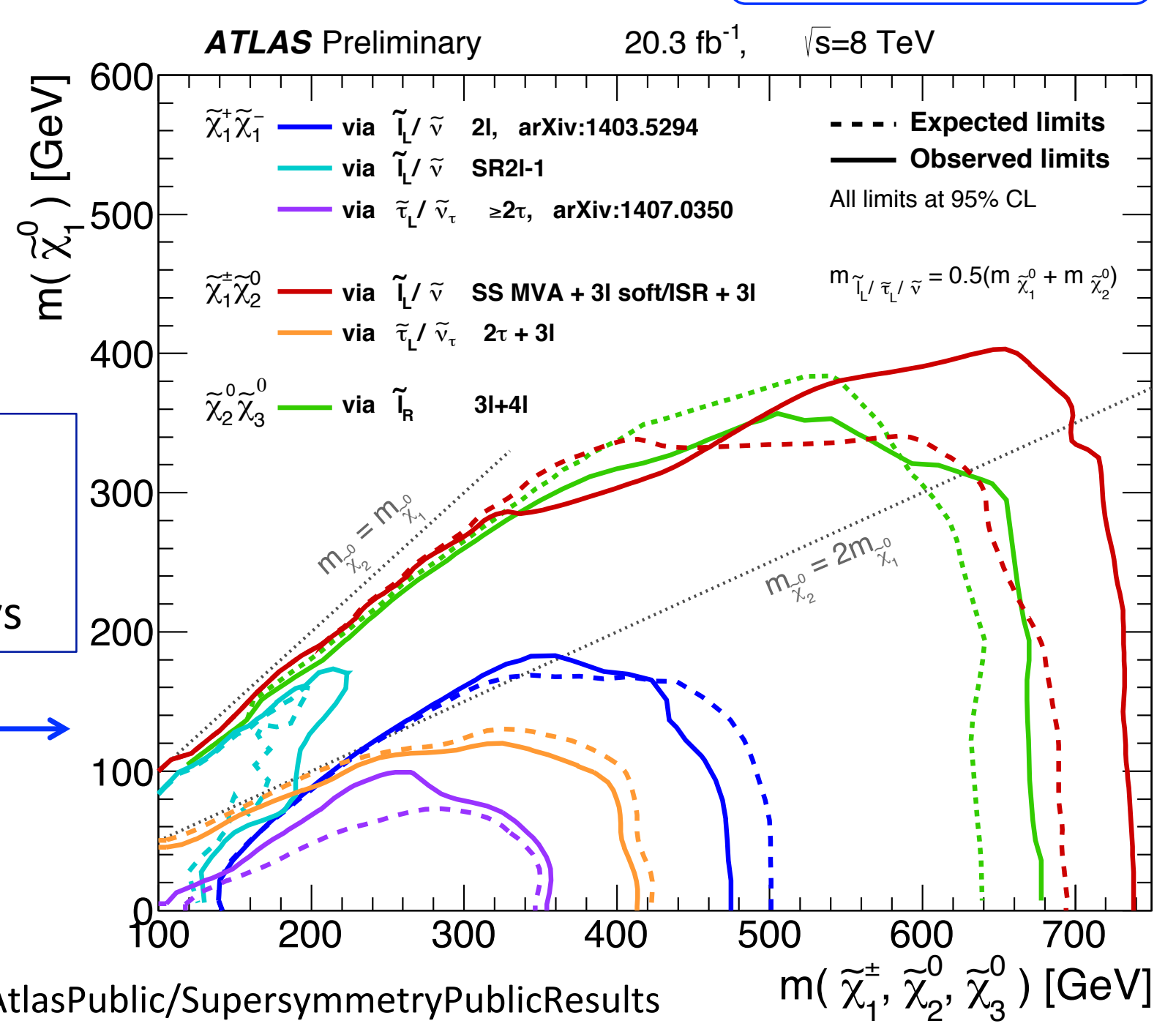




\section{Run1}

\section{Vs $=8 \mathrm{TeV}$}

\section{RPV Searches}

- Dedicated searches probing RPV signatures +

- + re-interpretations of RPC searches

- Only a few examples shown in this talk 


\section{Run1 SUSY Searches in Summary}




\section{Run1 SUSY Searches in Summary}

- 19 dimensional phenomenological MSSM (pMSSM) sampled

- 310,000 models surviving all theory and non-LHC experimental constraints interpreted using the searches in 22 ATLAS run1 papers

- fraction of models excluded is shown

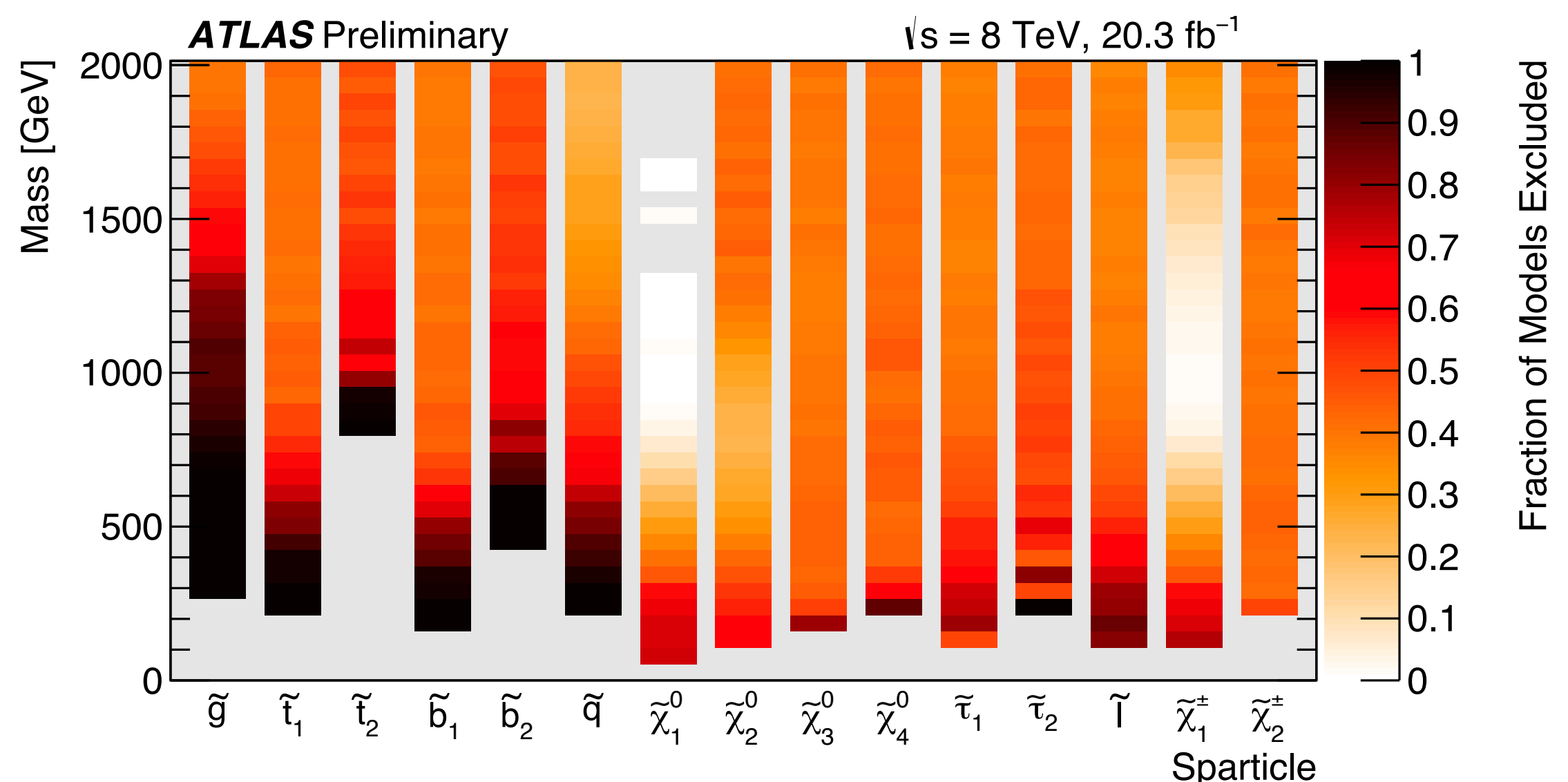




\section{What's next?}




\section{Prospects for RUN2}

\section{Estimated discovery reach at $13 \mathrm{TeV}$ for $1,2,5$ and $10 \mathrm{fb}^{-1}$}

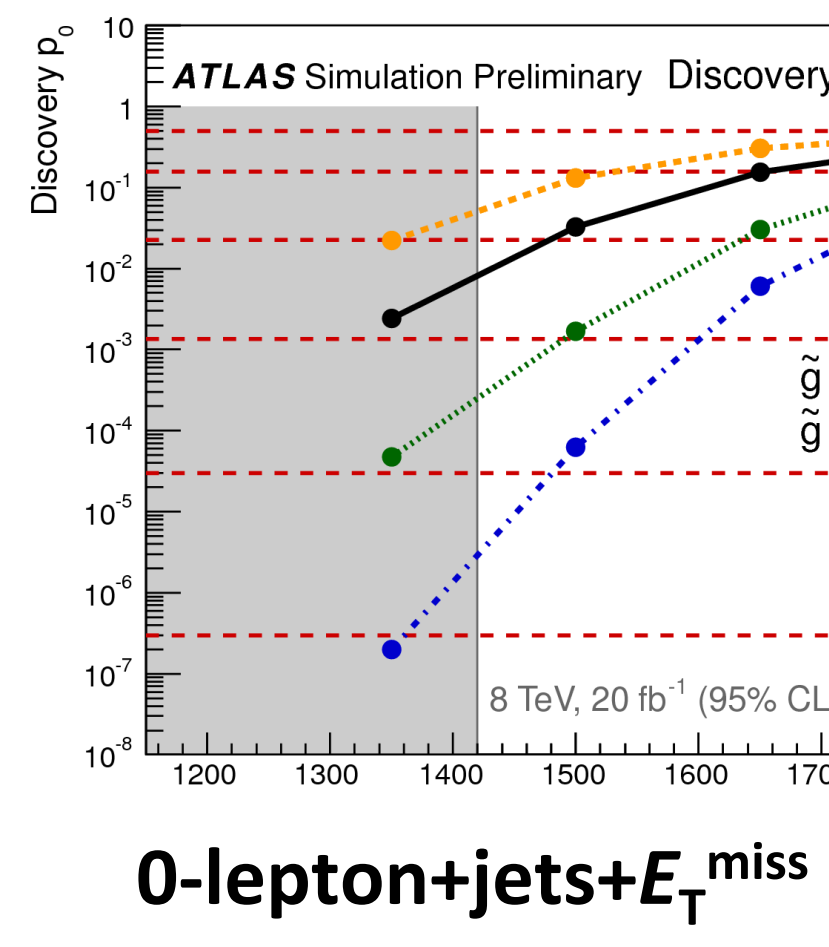

ATL-PHYS-PUB-2015-005

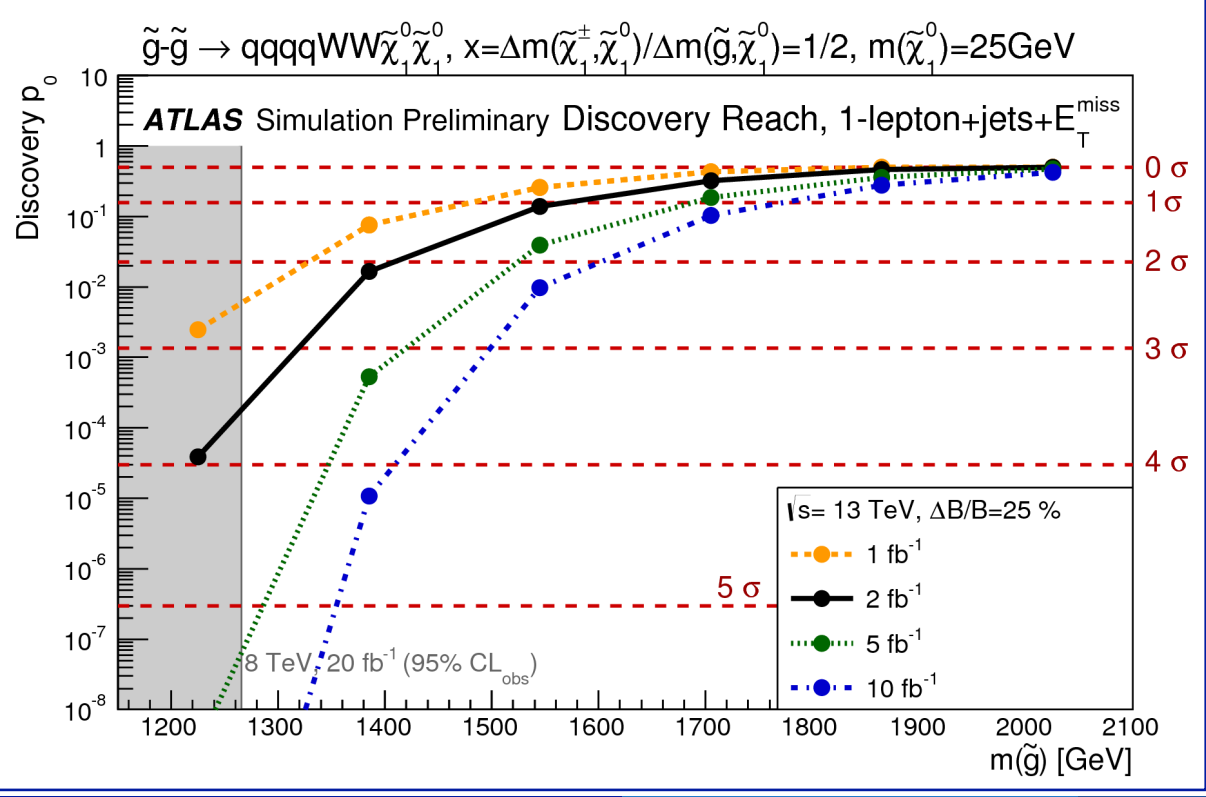




\section{A first look in RUN2}

- First data collected

- Performance assessment has started:

- E.g. Control Region distributions in strong production searches

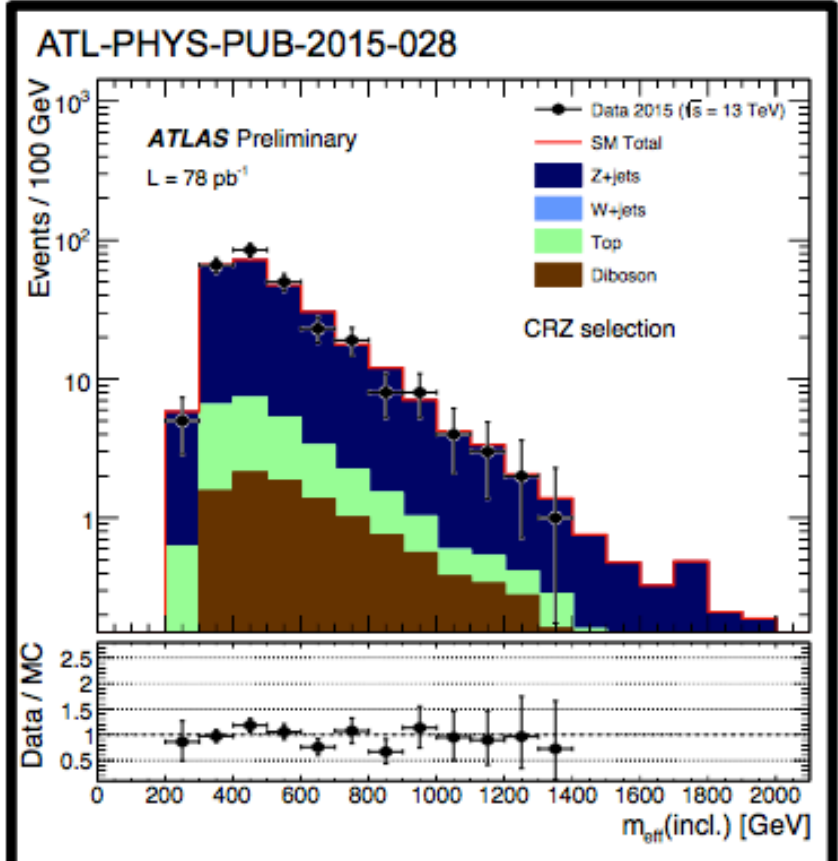

0-lepton (2-6 jets) + MET $\mathrm{m}_{\text {eff }}$ in " $\mathrm{Z}$ control region"

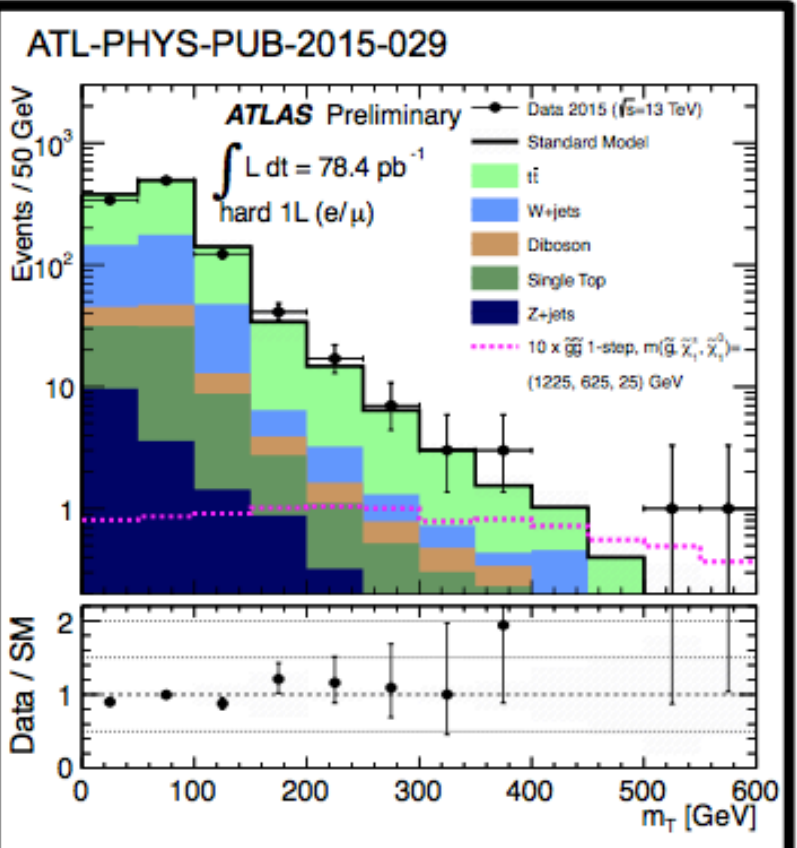

1-lepton (2-6 jets) + MET $\mathrm{m}_{\mathrm{T}}$ after loose selection on lepton + MET + 4-jet

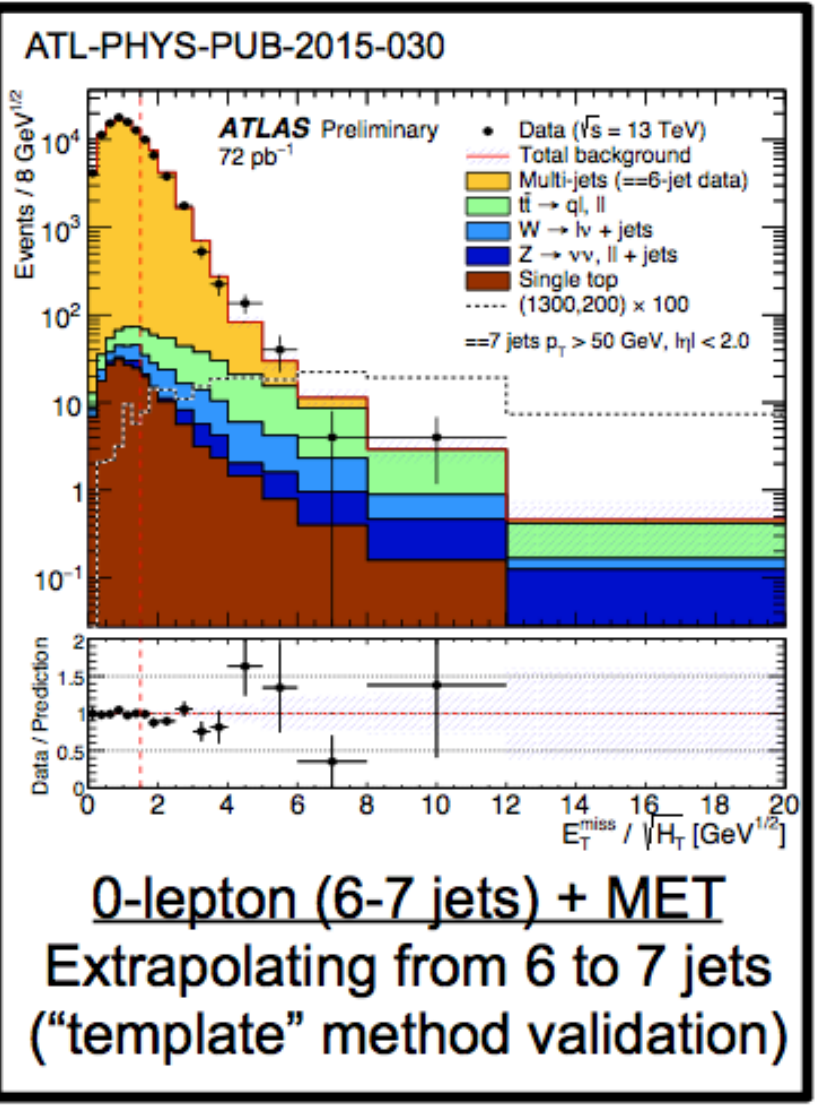

$17^{\text {th }}$ Lomonosov Conference on Elementary Particle Physics 


\section{Summary}

- Many different SUSY searches performed with Run1 data:

- targeting many production processes and

- covering a wide range of final states

- $95 \% \mathrm{CL}$ exclusion limits set for a variety of models but...

- 1 exciting excess observed in the Z+MET analysis

- Great potential in Run2...

- which has already started!! 
BACKUP 


\section{ATLAS SUSY Searches* - 95\% CL Lower Limits}

ATLAS Preliminary

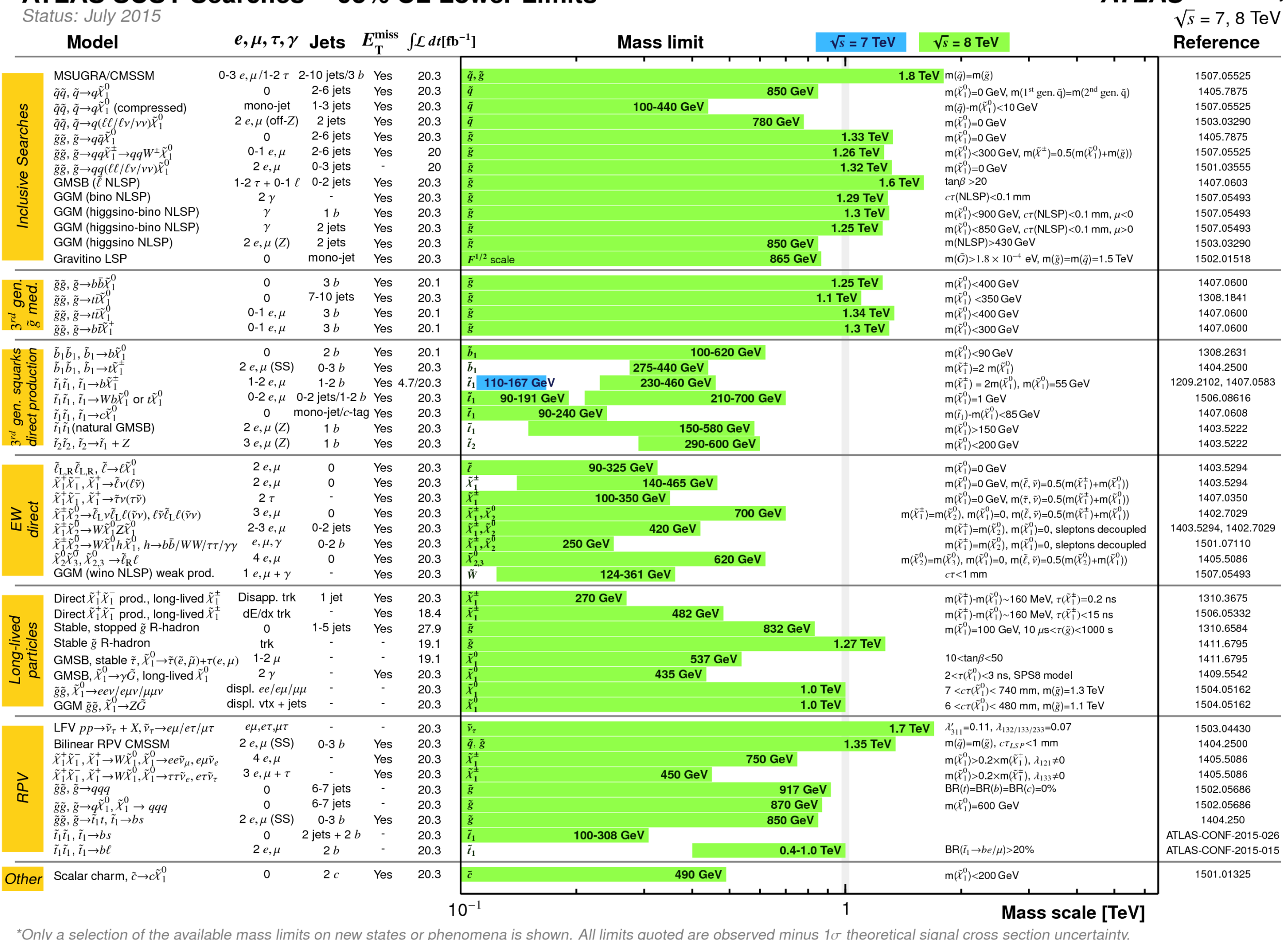

*Only a selection of the available mass limits on new states or phenomena is shown. All limits quoted are observed minus $1 \sigma$ theoretical signal cross section uncertainty. 


\section{Electroweak Production Searches}

- Small cross-sections but:

$V s=8 \mathrm{TeV}, \mathrm{L}=20 \mathrm{fb}^{-1}$

- Clean multi-lepton final states

- Low hadronic activity

- Searches using e, $\mu, \tau$

Summary plot showing 95\% CL exclusion limits obtained by different searches
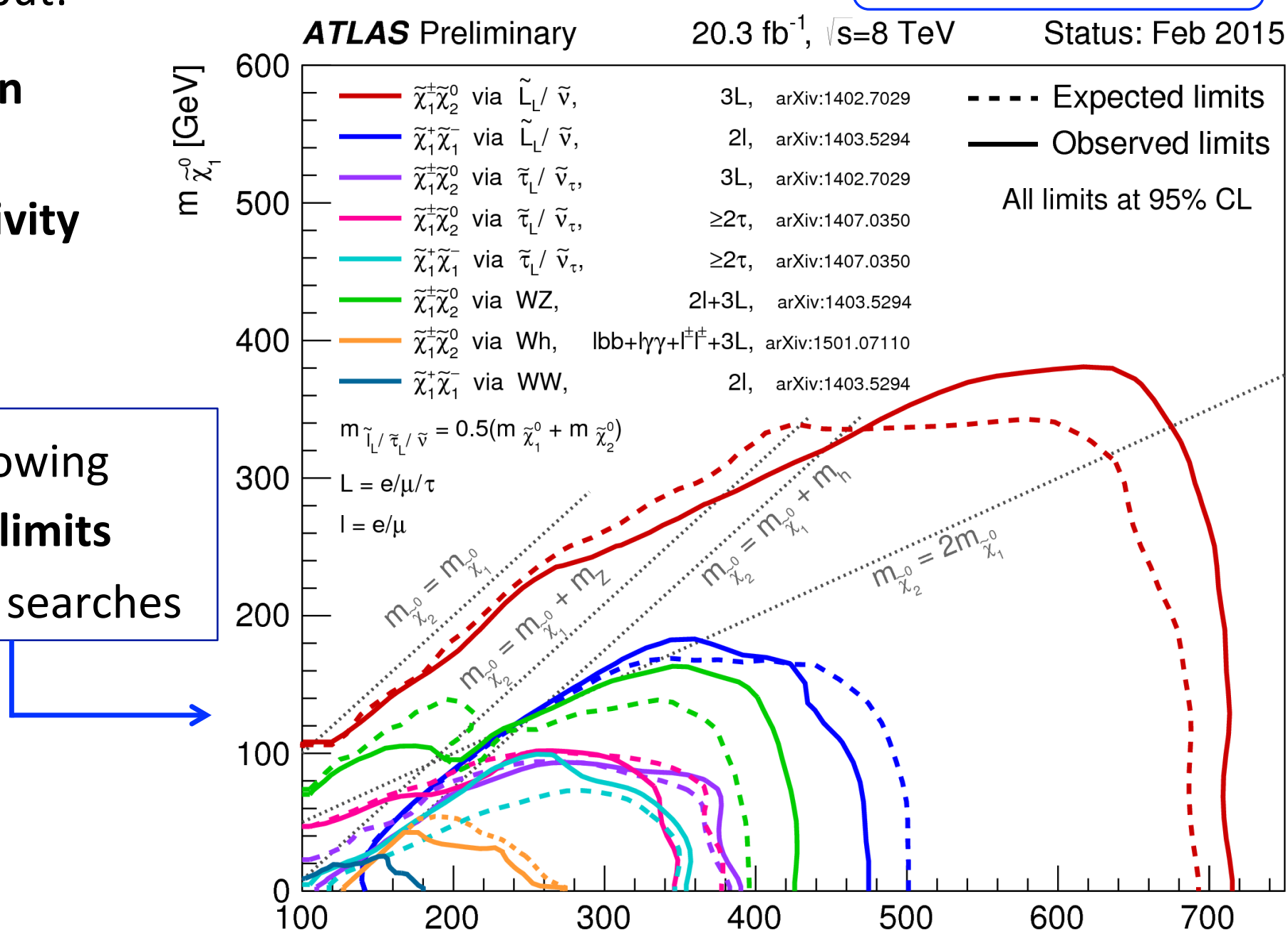

Publication references shown in the plot 


\section{Long-lived particles}

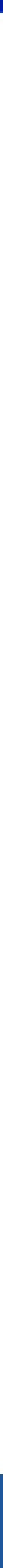




\section{ATLAS SUSY Search Strategy}

\section{SUSY events in our data?}

- Define Signal Region(s) (SR): set of cuts (on EtMiss, jet multiplicity, etc) that favour signal over background

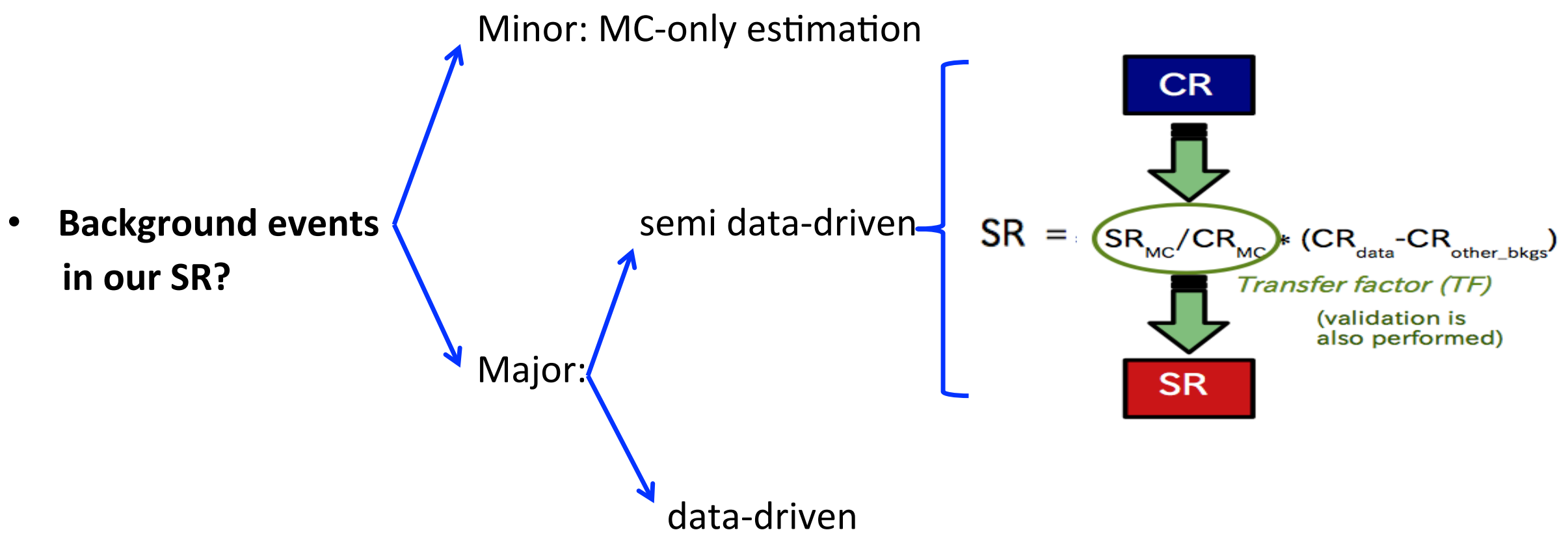

- Discovery/Exclusion fit:

- CLs exclusion limits or discovery 


\section{Classifying SUSY searches}

\section{Searches split by production process}

- Strong production

- targeting $1^{\text {st }}$ and $2^{\text {nd }}$ generation squarks and gluinos

- largest cross-sections

- $3^{\text {rd }}$ generation production

- targeting stops and sbottoms

- Electroweak production

- targeting:

- electroweakinos (charginos \& neutralinos)

- sleptons

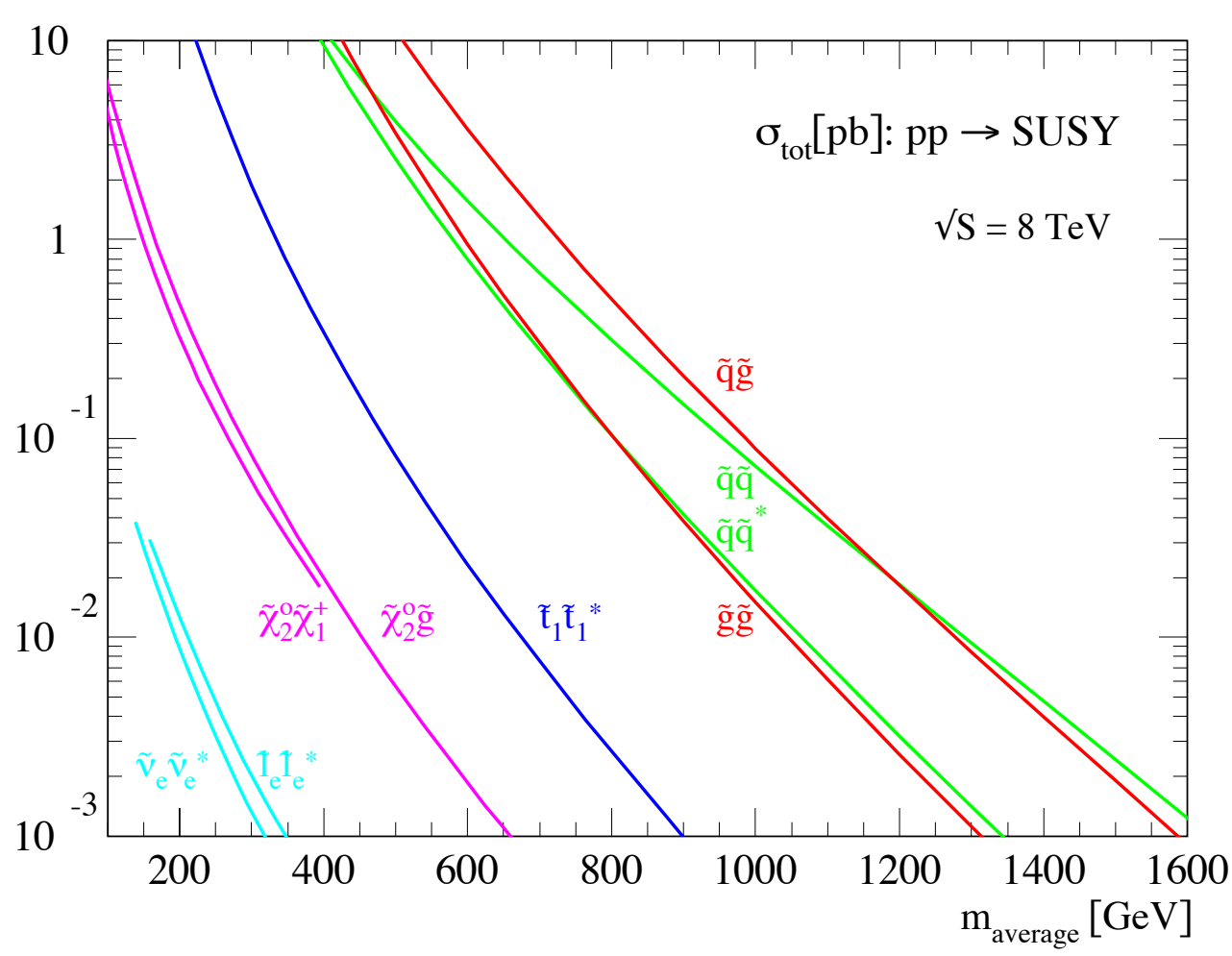

... and by final state characteristics

- R-parity conserving (RPC) SUSY: neutral stable LSP, sparticles produced in pairs, large MET

- R-parity violating (RPV) SUSY: LSP decays into SM particles => single sparticle production

- Long-Lived particles, within RPC and RPV 
mSUGRA/CMSSM Parameters

- $\rightarrow$ gravity-mediated SUSY breaking

- $m_{0}$ : mass of scalar particles

- $m_{1 / 2}$ : gaugino masses

- $A_{0}$ : trilinear Higgs-sfermion-sfermion coupling parameter

- $\tan \beta=\nu_{u} / \nu_{d}$ : ratio of the vacuum expectation values of the two Higgs doublets

- sign of the Higgsino mass parameter $\mu$

\section{GMSB Parameters}

- $\rightarrow$ gauge-mediated SUSY breaking

- $\Lambda$ : SUSY breaking mass scale felt by the low-energy sector

- $M_{\text {mes }}$ : mass scale of the messenger fields

- $N_{5}$ : number of SU(5) messenger fields

- $C_{\text {grav }}$ : scale factor of the gravitino coupling

- $\tan \beta=\nu_{u} / \nu_{d}$ : ratio of the vacuum expectation values of the two Higgs doublets

- sign of the Higgsino mass parameter $\mu$

\section{NGM}

- starts from General Gauge Mediation

- GGM: no specific SUSY mass hierarchy is predicted for colored and uncolored states $\Rightarrow$ gluinos and squarks can be below the TeV scale $=$ within reach of LHC

- NGM: decouple all sparticles not related to fine-tuning of Higgs sector $\Rightarrow$ light stop and light gluino as only light (relevant) coloured sparticle

- some additional mechanism needed (as in GMSB) to produce "correct" Higgs mass

Alexander Mann (LMU München)

Elena Romero Adam - IFIC
Strong SUSY Production at the LHC

(1)

$17^{\text {th }}$ Lomonosov Conference on Elementary Particle Physics
Moriond QCD - 11.03.2013

August 25, 2015 


\section{Target models}

- General Gauge Mediation (GGM)

- RPC model

- LSP always the gravitino (escapes detection $=>E_{T}{ }^{\text {miss }}$ )

- Phenomenology depends on NLSP (next to lightest SUSY particle): $\tilde{\chi}_{1}^{0}$ for most of the parameter space

- This analysis:

- $\tilde{\chi}_{1}^{0}$ higgsino-like

- Parameters: $M_{1}=M_{2}=1 T e V, c \tau_{N L S P}<0.1 \mathrm{~mm} ; m_{\text {gluino }}$ and $\mu$ : free parameters; all other sparticle masses $\approx 1.5$

- Two grids studied:

- $\tan \beta=1.5: \quad \tilde{\chi}_{1}^{0} \rightarrow Z \tilde{G}$

$-\tan \beta=30:\left\{\begin{array}{l}\tilde{\chi}_{1}^{0} \rightarrow Z \tilde{G} \\ \tilde{\chi}_{1}^{0} \rightarrow h \tilde{G}\end{array}\right.$

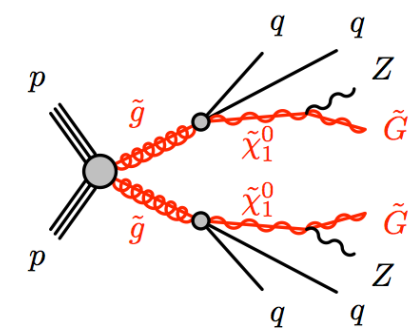

Elena Romero Adam - IFIC

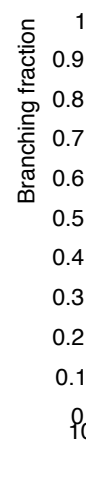

$\tan \beta=30$

$\tilde{\chi}_{1}^{0} \rightarrow \mathrm{Z} \tilde{\mathrm{G}}$
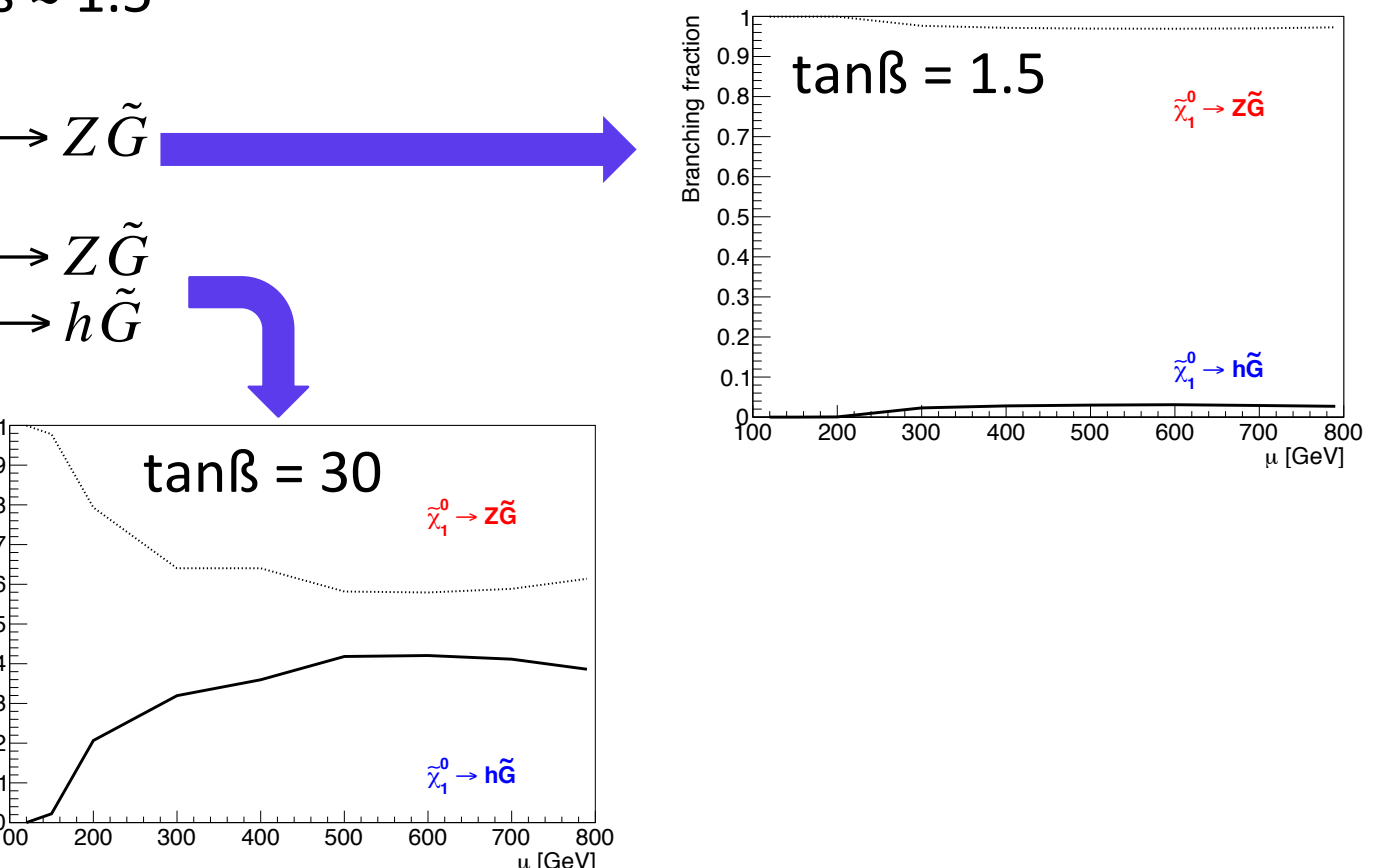


\section{Common object definitions}

Representative object definitions used by the ATLAS SUSY Working Group

1. Jets: Built from calorimeter clusters using the anti- $k_{t}$ association scheme with radius parameter $R=0.4$, and calibrated to correct for dead material, calorimeter response, pile-up etc. Analyses use jets with $|\eta|<2.8$ and varying thresholds on $p_{T}$ and the fraction of tracks originating from the primary vertex (JVF), whereas all jets with $|\eta|<4.9$ and $p_{T}>20 \mathrm{GeV}$ enter $Z_{T}$.

2. Muons: Identified as ID tracks combined with MS track segments, with $p_{T}>10 \mathrm{GeV}$ $\overline{\text { and }|\eta|}<2.4$. "Signal" muons have $p_{T}>20 \mathrm{GeV}$ and have higher object quality and isolation requirements.

3. Electrons: Identified as ID tracks combined with calorimeter clusters, with $p_{T}>20 \mathrm{GeV}$ and $|\eta|<2.47$. "Signal" electrons have $p_{T}>25 \mathrm{GeV}$ and have higher object quality and isolation requirements.

4. Photons: Identified on the basis of shower shape in the calorimeter or from conversion tracks, with $p_{T}>20 \mathrm{GeV},|\eta|<2.37$ and $(1.52<|\eta|$ or $1.37>|\eta|)$. Additional "ambiguity resolution" criteria reduce contamination from electrons. A transverse energy isolation requirement of $<5 \mathrm{GeV}$ is imposed in a narrow cone of $\Delta R<0.2$.

5. Tau jets: Identified using a multivariate discriminator (BDT) taking into account track information and calorimeter shower shapes, with $p_{T}>20 \mathrm{GeV},|\eta|<2.5$ and containing 1 or 3 tracks of $p_{T}>1 \mathrm{GeV}$ and with a charge sum of \pm 1 .

6. $b$-jets: Identified using multivariate discriminators taking into account impact parameter and secondary vertex information. 


\section{Background Estimation}

\section{$\underline{Z}+$ jets background} Jet smearing method:

\section{SM backgrounds:}

- QCD and inclusive W+jets

- $\mathrm{WZ}, \mathrm{ZZ}$

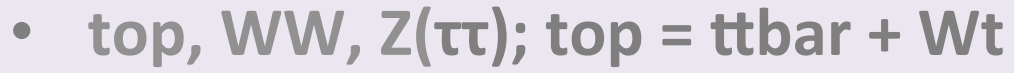

- $\mathrm{Z}+$ jets

Signal: $Z+$ jets + EtMiss $\longrightarrow$ from LSP

(Instrumental) bkg: Z + jets + fake Etmiss

from mis-measurement of jets

Estimating number of events in SR with high fake Etmiss $\measuredangle$ Estimating of number of $Z+$ jets bkg events in SR

How do we estimate the number of events with high fake EtMiss?

SEED REGION

region with well

measured jets $=>$ low

EtMiss region $\times$ RESPONSE FUNCTION=

modeling the

response of the

calorimeters
PSEUDO-DATA

simulation of events with no real EtMiss after passing throught the calorimeter

Applying SR cuts to pseudo-data $=>$ Estimation of number of $Z+$ jets bkg events in SR 


\section{bRPV Phenomenology}

- Model that we consider:

- $\quad$ LSP = lightest neutralino

- minimal Supergravity (mSUGRA): 5 parameters

- $\mathrm{m0}, \mathrm{m1} / 2$ : scalar and gaugino masses at GUT scale

- A0: trilinear scalar coupling

- $\tan \beta$ : ratio of Higgs vacuum expectation values

- $\operatorname{sign}(\mu)$ : sign of the higgsino mass parameter 


\section{bRPV}

- R-Parity is violated through bilinear terms in the superpotential:

- The new terms in $W_{\mathrm{bRPV}}=W_{\mathrm{MSSM}}+\epsilon_{i} L_{i} H_{u}$ itrinos and neutralinos:

- Explanation for neutrino masses and mixings

- Connection between neutrino parameters and neutralino decays:

- Hence, bRPV ${ }^{\tan ^{2} \theta_{23}} \approx \frac{\mathrm{BR}\left(\widetilde{\chi}_{1}^{0} \rightarrow \mu^{ \pm}+W^{\mp}\right)}{\operatorname{BR}\left(\widetilde{\chi}_{1}^{0} \rightarrow \tau^{ \pm}+W^{\mp}\right)}$ to be consistent with constraints from neutrino experiments 


\section{Results}

yellow band $\rightarrow 1 \sigma$ statistical and systematic uncertainties on the expected background

\section{dashed red lines $\rightarrow$ influence of the} theoretical uncertainties on the signal cross section on the limit 